\title{
Автоматизация метода реконструкции неотектонических напряжений Л.А. Сим с применением алгоритмов компьютерного зрения
}

Молчанов А.Б., Гордеев Н.А.

ИФ3 РАН, Москва, alexeybm2009@gmail.com; gord@ifz.ru

Аннотация. Ежегодно возрастает необходимость в качественном, быстром и надёжном определении напряженного состояния самых разных геологических объектов. Причина этому - возрастающая потребность в ископаемых ресурсах и заселении все новых участков земли.

На протяжении нескольких лет ведется активная разработка программного пакета Stress-analysis, в который входит ряд креативных решений, написанных на Python и Fortran для анализа напряженного состояния геологических структур разного масштаба.

В представленной работе обсуждается качество работы программного обеспечения SimSGM (Гордеев и др., 2019). В этом приложении автоматизирован структурно-геоморфологический метод реконструкции неотектонических напряжений Сим Л.А. (Сим, 1991), основанный на дешифрировании так называемых мегатрещин (прямолинейных элементов рельефа), по их характерному расположению в области разрыва или ослабленной зоны детально устанавливается напряженное состояние и кинематика разрывов или ослабленных зон.

Сопоставление результатов ручного и компьютерного методов показало высокую степень доверия, так как при независимом их использовании получились схожие реконструкции (свыше 85 \%).

Ключевые слова: SimSGM, Руthon, структурно-геоморфологический метод, неотектоника.

\section{Application of computer vision algorithms in tectonophysics}

Molchanov A.B., Gordeev N.A.

IPERAS, Moscow, alexeybm2009@gmail.com; gord@ifz.ru

\begin{abstract}
The need for high-quality, fast and reliable determination of the stress state of various geological objects is growing every year. The reason for this is the growing need for fossil resources and the settlement of more and more land plots.

For several years, the Stress-analysis software package has been actively developed, which includes a number of creative solutions written in Python and Fortran for analyzing the stress state of geological structures at various scales.

The presented work discusses the advantages of the SimSGM software (Gordeev et al., 2019). This application automates the structural-geomorphological of L.A. Sim neotectonic stresses reconstruction method. (Sim, 1991), which is based on the interpretation of the so-called megafracks (rectilinear relief elements), the stress state and kinematics of ruptures or weakened zones are established in detail by their characteristic location in the area of a rupture or weakened zone.

The results comparison of manual and computer methods showed a high degree of confidence, since their independent use resulted in similar reconstructions (over $85 \%$ ).
\end{abstract}

Key words: SimSGM, Python, structural-geomorphological method, neotectonics.

\section{Введение}

В настоящее время происходит активное развитие алгоритмов компьютерного зрения, а область их применения с каждым днём становится всё шире. К текущему моменту известно множество алгоритмов, используемых для анализа изображений с целью поиска на них особенностей определённого рода. Наиболее популярен ряд алгоритмов, основанных на искусственных нейронных сетях, поскольку такого рода алгоритмы позволяют выделять на изображениях особенности, определяемые нейросетью самостоятельно на основе обучения.

В данной работе будет идти речь о применении технологий компьютерного зрения к спутниковым снимкам и картам высот. При этом основными особенностями будут являться линейные элементы рельефа. Это и некоторые другие обстоятельства позволяют считать применение нейросетевых алгоритмов избыточным в настоящем исследовании, поскольку параллельно развиваются и 
классические алгоритмы, направленные на поиск на изображении особенностей, форма которых задаётся напрямую пользователем. Этот класс алгоритмов проще в реализации и, как будет показано в данной работе, демонстрирует уверенно верифицируемые результаты.

Целью применения алгоритмов компьютерного зрения в настоящей работе является получение цифрового представления линейных элементов рельефа, интерпретируемых как трещины, с последующим восстановлением по их конфигурации направления осей тектонических напряжений в областях динамического влияния геологических разломов. Восстановление ориентации тектонических напряжений происходит по методике, разработанной в лаборатории Тектонофизики ИФЗ РАН.

\section{Описание методики}

Метод реконструкции сдвиговых тектонических напряжений (Сим, 1991) (структурногеоморфологический метод) в своей основе имеет анализ закономерности распределения оперяющих разрывов в зоне динамического влияния сдвиговых разрывов и ослабленных зон. Впервые такие закономерности обобщил М.В. Гзовский (1975). Основным рабочим материалом в методе являются данные дешифрирования линеаментов, которые в исследовании имеют свое собственное название - мегатрещины, так как являются косвенным признаком тектонической активности разрывов и ослабленных зон. Если взаиморасположение мегатрещин соответствует одному из вариантов парагенезиса оперяющих трещин в зоне сдвига (рис. 1), то исследователю удается определить направление сдвига и ориентировки горизонтальных осей сжатия и растяжения с осложняющими обстановками транспрессии или транстенсии в локальной точке, т.е. определить кинематику и локальное стресс-состояние. Возраст реконструируемых напряжений считается новейшим или современным по причине выраженности мегатрещин в новейших и четвертичных образованиях.

В приложении SimSGM все алгоритмы написаны на языке программирования Python. Программа работает на OC Windows c разрядностью 64 бит. В работе программы выделяется три основных этапа:

- Загрузка цифровой модели рельефа, космо-снимка;

- Дешифрирование линеаментов;

- Анализ напряженного состояния с классификацией по М.В. Гзовскому (1975) (рис. 1).

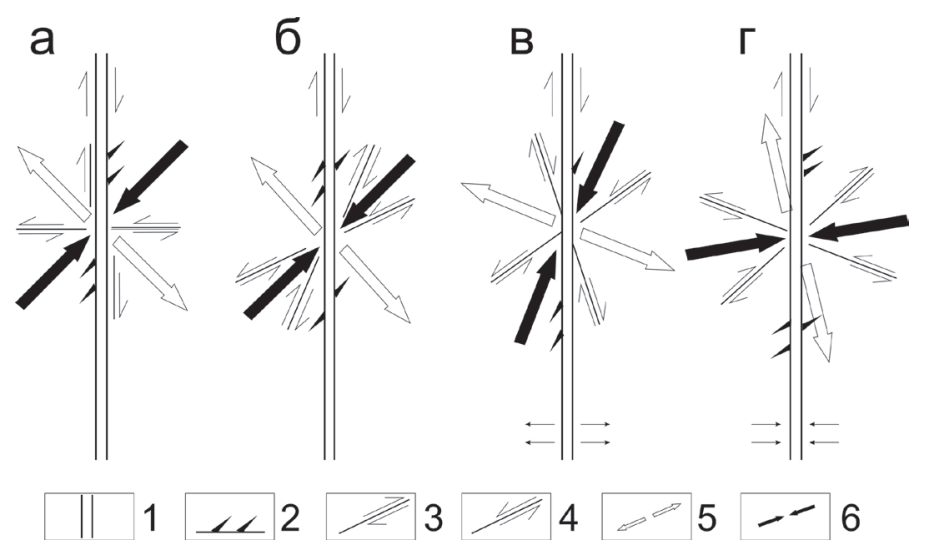

Рис. 1. Парагенезис оперяющих трещин в зоне сдвига (Гзовский, 1975), или «палетка Гзовского». Варианты напряженного состояния при углах скалывания: близких к $45^{\circ}(\mathrm{a}),<45^{\circ}$ (б); обстановки дополнительного растяжения (в) и сжатия (г) - показаны в нижней части разломов стрелками, нормальными к плоскости разлома. 1 - разлом; 2 - трещина отрыва; 3, 4 - сколы с правой (3) и левой (4) сдвиговой кинематикой; 5, 6 - ориентация осей растяжения (5) и сжатия (6) в горизонтальной плоскости.

Fig. 1. Paragenesis of feathering cracks in the shear zone (Gzovsky, 1975), or «Gzovsky's palette». Variants of the stress state at shear angles: close to $45^{\circ}(\mathrm{a}),<45^{\circ}$ (b); settings of additional extension (c) and compression (d) are shown in the lower part of the faults by arrows normal to the fault plane. 1 - fault; 2 - tear-off crack; 3, 4-chips from the right (3) and left (4) shear kinematics; 5, 6 - orientation of the axes of extension (5) and compression (6) in the horizontal plane. 
На усмотрение пользователя есть возможность полуавтоматического или полностью автоматического анализа. В первом случае пользователь проводит разрыв и отмечает мегатрещины, во втором оператору нужно провести разрыв/ослабленную зону (оставляя остальное для алгоритмов компьютерного зрения).

Из удобных элементов программы для полуавтоматического способа анализа - реализована возможность подгрузки полностью готовой схемы разрывов с прилегающими к ним мегатрещинам в формате SVG. На этапе классификации все происходит в режиме реального времени. Вдоль разрыва отображается скользящее масштабируемое окно, в области которого анализируются все попадающие в него мегатрещины.

Для работы в автоматическом режиме необходимо загрузить карты высот или спутниковый снимок. Разрыв в этом случае проводится по методу «активного контура» (Kass, 1988), реализованного в библиотеке Sci-kit Image. Между указанными началом и концом разрыва создаётся сплайн, стремящийся к затемненным участкам карты высот или карты когерентности.

В автоматическом режиме применяются методы компьютерного зрения к бинаризованному изображению загруженных данных. Карты высот преобразуются в «скелетное» изображение главных понижений, проявленных в эрозионной сети и других резких перепадах высот (Гордеев и др., 2019). Спутниковые карты преобразуются в карты когерентности, т.е. снимок переводится в оттенки серого, где обратная интенсивность обозначает неоднородность изображения. Далее так же производится скелетизация. На финальном этапе следует анализ с классификацией по М.В. Гзовскому (1975) (рис. 1).

Классификация проводится по цепи условий, по которым проходит проверка каждого отдельного угла между разрывом и мегатрещиной с учётом степени кривизны выбранной мегатрещины (Гордеев и др., 2019).

\section{Выводы}

Верификация проводилась на территории Лено-Оленекского междуречья с контрастным рельефом, где есть области малоамплитудного рельефа дельты р. Лены, высокоамплитудного север-

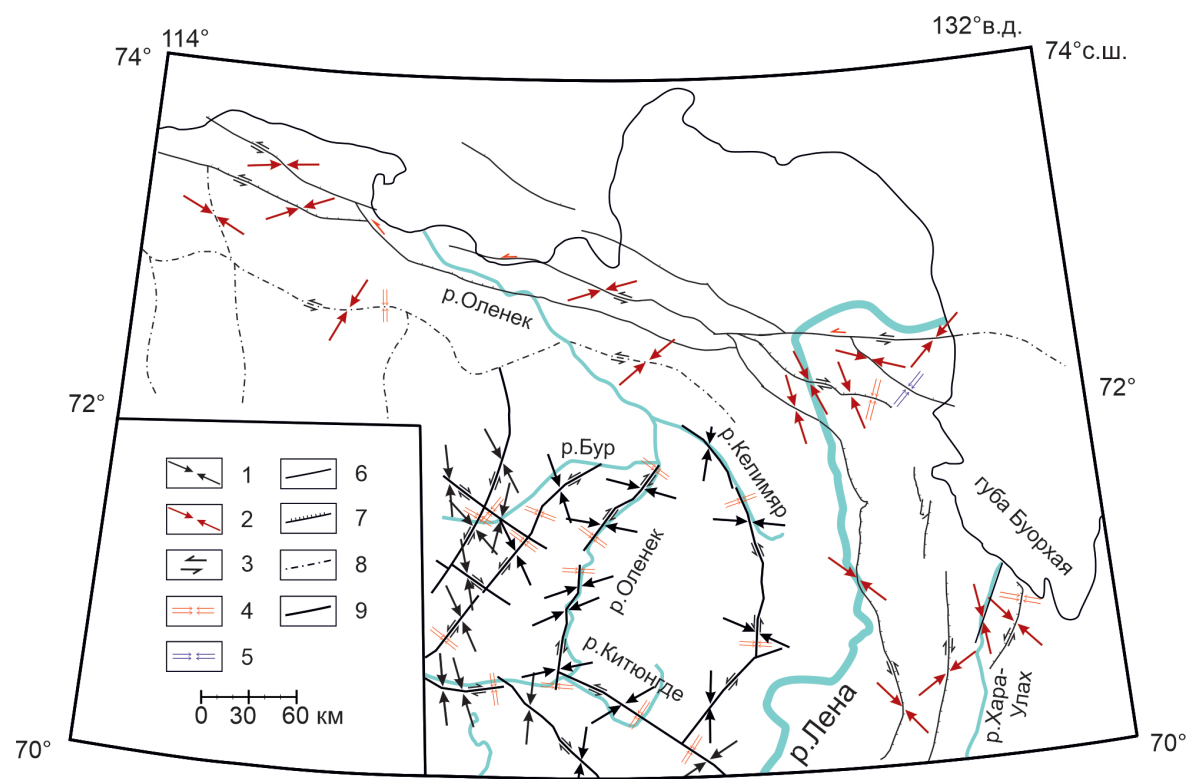

Рис. 2. Результаты обработки ручным методом реконструкции сдвиговых тектонических напряжений. 1-результаты реконструкций ручным методом (Гордеев, 2019); 2 - результаты реконструкций ручным методом (новые); 3 - направления сдвигов; 4 - условия транспрессии; 5 - условия транстенсии; 6 - разлом второго ранга; 7 - разлом (надвиг); 8 - погребенный разлом; 9 - разлом первого ранга.

Fig. 2. Results of processing by manual method of shear tectonic stresses reconstruction. 1 - results of manual reconstructions (Gordeev, 2019); 2 - results of manual reconstructions (new); 3 - direction of shifts; 4 - conditions of transpression; 5 - conditions of transtension; 6 - fault of the second rank; 7 - fault (thrust); 8 - buried fault; 9 - first rank fault. 


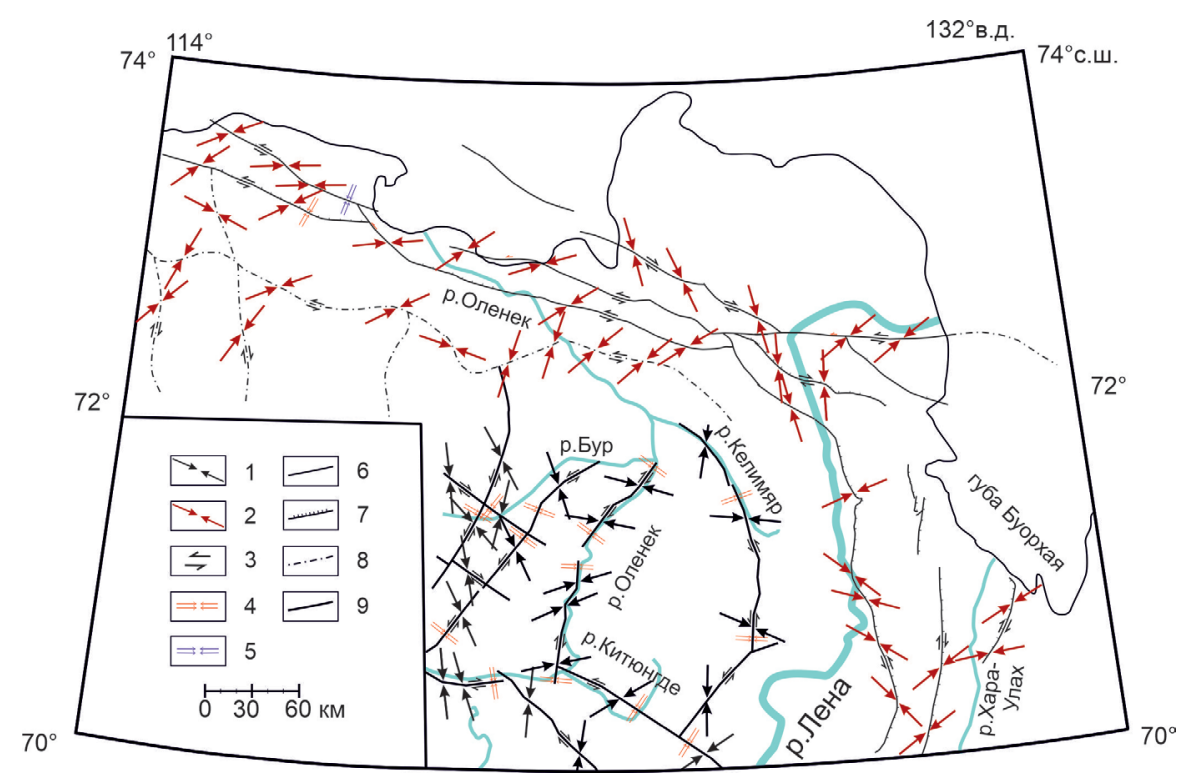

Рис. 3. Результаты обработки программой SimSGM. 1 - результаты реконструкций ручным методом (Гордеев, 2019); 2-результаты реконструкций программой (новые); 3 - направления сдвигов; 4 - условия транспрессии; 5 - условия транстенсии; 6 - разлом второго ранга; 7 - разлом (надвиг); 8 - погребенный разлом; 9 - разлом первого ранга.

Fig. 3. Results of processing by the SimSGM program. 1 - results of manual reconstructions (Gordeev, 2019); 2 - results of reconstruction by the program (new); 3 - direction of shifts; 4 - conditions of transpression; 5 - conditions of transtension; 6 - fault of the second rank; 7 - fault (thrust); 8 - buried fault; 9 - first rank fault.

ного окончания Верхоянского хребта и Кряжа Чекановского, общие данные по контрасту рельефа от первых метров до свыше 1000 м.

Реконструированные локальные стресс состояния без применения SimSGM (рис. 2) на 85 \% совпадают с обработкой программой (рис. 3). Подобный результат говорит о том, что применяемые методы компьютерного зрения обеспечивают уверенный анализ как на слаборасчлененных территориях, так и на контрастном рельефе, а статистическая обработка программой позволяет анализировать куда больший объем данных по мегатрещинам, чем это может себе позволить человек. Высокое совпадение результатов ручного и компьютерного анализа позволяет сделать вывод, что применение методов компьютерного зрения оправдано по отношению к неотектонической интерпретации новейшей тектоники методом реконструкции сдвиговых неотектонических напряжений Сим Л.А. (1991).

\section{Литература}

1. Гордеев Н.А., Молчанов А.Б. Автоматизация структурно-геоморфологического метода реконструкции сдвиговых неотектонических напряжений Л.А. Сим // Геоинформатика. 2019. № 2. С. $25-33$.

2. $\quad$ Гзовский М.В. Основы тектонофизики. М. Изд-во: Наука. 1975. 375 с.

3. Сим Л.А. Изучение тектонических напряжений по геологическим индикаторам (методы, результаты, рекомендации) // Изв. ВУЗов. геол. и разв. 1991. № 10. С. 3-22.

4. Kass M., et.al. Snakes:Active contour models // International Journal of Computer Vision 1 (4): 321 (1988). 